\title{
Calligonum comosum extract inhibits diethylnitrosamine-induced hepatocarcinogenesis in rats
}

\author{
WALIED ABDO ${ }^{1,2}$, AKIHIRO HIRATA $^{3}$, MOSTAFA SHUKRY $^{4}$, TAREK KAMAL ${ }^{5}$, \\ ESSAM ABDEL-SATTAR $^{6}$, ENGI MAHROUS ${ }^{6}$ and TOKUMA YANAI ${ }^{2}$
}

\author{
${ }^{1}$ Department of Pathology, Faculty of Veterinary Medicine, Kafrelsheikh University, Kafr el-Sheikh 33516, Egypt; \\ ${ }^{2}$ Laboratory of Veterinary Pathology, Department of Veterinary Medicine, Faculty of Applied Biological Sciences; \\ ${ }^{3}$ Division of Animal Experiment, Life Science Research Center, Gifu University, Gifu 501-1193, Japan; \\ Departments of ${ }^{4}$ Physiology and ${ }^{5}$ Biochemistry, Faculty of Veterinary Medicine, Kafrelsheikh University, \\ Kafr el-Sheikh 33516; ${ }^{6}$ Department of Pharmacognosy, Faculty of Pharmacy, Cairo University, Cairo 11562, Egypt
}

Received June 4, 2014; Accepted February 23, 2015

DOI: $10.3892 / 01.2015 .3313$

\begin{abstract}
Calligonum comosum (C. comosum) is an Egyptian desert plant that contains polyphenol antioxidants. The present study examined the chemopreventive effect of an extract of C. comosum in a rat model of hepatocarcinogenesis. Male Wistar rats $(\mathrm{n}=40)$ were administered $100 \mathrm{mg} / \mathrm{kg}$ diethylnitrosamine (DEN) by intraperitoneal (i.p.) injection once a week for 3 weeks. Subsequently, depending on whether the rats received further administration of $0.8 \mathrm{mg} / \mathrm{kg}$ carbon tetrachloride $\left(\mathrm{CCl}_{4}\right)$ i.p. once a week for 7 weeks and $100 \mathrm{mg} / \mathrm{kg}$ C. comosum extract in their diet for 7 weeks, the rats were divided into four groups as follows: Group 1, treatment with DEN alone; group 2, treatment with DEN and C. comosum extract; group 3, treatment with DEN and $\mathrm{CCl}_{4}$; and group 4, treatment with $\mathrm{DEN}, \mathrm{CCl}_{4}$ and C. comosum extract. The supplementation of $C$. comosum extract significantly suppressed the elevation in serum liver enzyme levels, including aspartate aminotransferase, alanine transaminase and $\gamma$-glutamyl transferase, and reduced the degree of oval cell proliferation induced by DEN and $\mathrm{CCl}_{4}$. In addition, C. comosum extract significantly decreased the number and area of glutathione S-transferase placental form-positive preneoplastic hepatic foci induced by DEN, with or without $\mathrm{CCl}_{4}$ treatment. To the
\end{abstract}

Correspondence to: Dr Walied Abdo, Department of Pathology, Faculty of Veterinary Medicine, Kafrelsheikh University, El-Giesh Street, Kafr el-Sheikh 33516, Egypt

E-mail: waliedsobhy@yahoo.com

Abbreviations: ALT, alanine aminotransferase; AST, aspartate aminotransferase; $\mathrm{CCl}_{4}$, carbon tetrachloride; DEN, diethylnitrosamine; DPPH, 1,1-diphenyl-2-picrylhydrazyl; GGT, gamma-glutamyltransferase; GST-P, glutathione S-transferase placental form; HCC, hepatocellular carcinoma; HPLC, high-performance liquid chromatography

Key words: Calligonum comosum, diethylnitrosamine, liver, rat, glutathione S-transferase placental form, oval cell proliferation best of our knowledge, the present study is the first to provide definitive evidence of the hepatoprotective and chemopreventive effects of $C$. comosum.

\section{Introduction}

Hepatocellular carcinoma (HCC) is one of the most prevalent malignancies in humans, with high cancer-associated mortality rates (1). Prominent risk factors associated with the development of HCC include chronic alcohol consumption, the ingestion of aflatoxin B1-contaminated food and hepatitis $\mathrm{B}$ and $\mathrm{C}$ viral infections (1). The incidence of HCC among geographical populations tends to reflect the local prevalence of its etiological risk factors. For instance, a high rate of HCC is observed in Egypt, where there is a high prevalence of hepatitis B and C viral infections (2). The relatively high exposure to aflatoxin B1 and pesticides in Egypt are also suspected to contribute to the high prevalence of HCC (2). Since it can be challenging to rapidly eliminate risk factors, the application of HCC chemopreventive therapy, particularly in carriers of the hepatitis B and C viruses, has clear benefits (3). Locally available plants may provide accessible and economically feasible sources of herbal HCC preventives.

Calligonum comosum (C. comosum) is an Egyptian desert plant that belongs to the Polygonaceae family and is known locally as 'arta'. C. comosum is used as a smokeless source of firewood and for tanning leather. In addition, it is used in traditional folkloric medicine to treat abdominal ailments and toothache (4). Several studies have demonstrated a number of biological effects of $C$. comosum extract in animal models, including anti-inflammatory activities, antigastric ulcer properties (5) and antihyperglycemic activities in rats (6). Furthermore, C. comosum exhibited anticancer activities in mice inoculated with Ehrlich ascites carcinoma cells (7). The biological properties of $C$. comosum may be associated with its antioxidative activity, which has been consistently demonstrated in vitro $(7,8)$. In accordance with this hypothesis, several antioxidative flavonoids have been isolated from the ethyl acetate fraction of $C$. comosum methanolic extract, including (+)-catechin, dehydrodicatechin A, kaempferol-3-O-rhamnopyranoside, 
quercetin (quercetin-3-O-rhamnopyranoside), isoquercitrin (quercetin-3-O-glucopyranoside),kaempferol-3-O-glucuronide and mequilianin (quercetin-3-O-glucuronide) (7). The antioxidative activity of the $C$. comosum extract has also been demonstrated in vivo. In particular, the extract has been identified to suppress the accumulation of malondialdehyde, one of the final decomposition products of lipid peroxidation, in brain and liver tissues of haloperidol-treated rats (8). On the basis of these findings, the present study aimed to investigate whether the $C$. comosum extract may reduce the formation of glutathione S-transferase placental form-positive (GST-P) preneoplastic hepatic foci in rats exposed to diethylnitrosamine (DEN), with or without administration of carbon tetrachloride $\left(\mathrm{CCl}_{4}\right)$. In addition, the current study evaluated whether the extract may be a potential chemopreventive agent in hepatocarcinogenesis.

\section{Materials and methods}

Plant materials and extract preparation. Aerial parts of C. comosum were obtained from Cairo-Suez Road (Cairo, Egypt) in April 2012. A herbarium specimen of the plant was identified by Dr. Mohamed Abdel-Aleem (Department of Botany, Faculty of Science, Cairo University, Cairo, Egypt), and a sample was kept in the Herbarium of the Department of Pharmacognosy (Faculty of Pharmacy, Cairo University; specimen number, CC-01204). The plant materials were air-dried, ground to a powder and then kept in dark, airtight, closed containers prior to extraction. In order to prepare the extract, a 750-g sample was extracted with three 2-liter volumes of absolute methanol in an Ultra Turrax T25 homogenizer (IKA ${ }^{\circledR}$-Werke GmbH \& Co. KG, Staufen, Germany). The solvent was then distilled under reduced pressure and the extracts were lyophilized, yielding a 69-g dry extract that was stored at $4^{\circ} \mathrm{C}$.

Phytochemical analysis. The phenolic content of the C. comosum extract was measured using the Folin-Ciocalteu method (9) with the modifications proposed by Abdel-Sattar et al (8). The total phenolic content was determined by measuring the absorbance at $685 \mathrm{~nm}$ using gallic acid as a reference standard (0.02-0.30 mg/ml) and the result was expressed in milligram equivalents of gallic acid per gram of dry extract. In addition, the total flavonoid content in the extract was determined using a previously described aluminum chloride colorimetric assay, which relies on quantifying the formation of flavonoid-aluminum complexes by measuring the absorbance at $430 \mathrm{~nm}$ (10). Quercetin was used as a reference standard $(0.03-0.30 \mathrm{mg} / \mathrm{ml})$. The total flavonoid content was expressed in milligram equivalents of quercetin per gram of dry extract.

Determination of 1,1-diphenyl-2-picrylhydrazyl (DPPH) radical-scavenging activity. The free radical-scavenging activity of the methanolic extract was measured based on its ability to quench the free radical-producing DPPH using a previously reported method $(8,11)$. Briefly, the concentration of DPPH was determined by measuring the absorbance at $517 \mathrm{~nm}$ prior to and subsequent to the addition of a specified quantity of the extract. Next, the $\%$ inhibition of radical formation was calculated using the following equation: $\%$ inhibition $=\left(\mathrm{A}_{\text {control }}-\mathrm{A}_{\text {sample }}\right) / \mathrm{A}_{\text {control }} \times 100$, where $\mathrm{A}_{\text {control }}$ is the absorbance of DPPH alone and $\mathrm{A}_{\text {sample }}$ is the absorbance of DPPH with $C$. comosum extract.

High-performance liquid chromatography (HPLC) analysis of C. comosum extract. In total, $100 \mathrm{mg}$ dried methanolic extract was hydrolyzed in $30 \mathrm{ml} 1.2 \mathrm{M} \mathrm{HCl}$ in $50 \%$ aqueous methanol at $80^{\circ} \mathrm{C}$ for $2 \mathrm{~h}$ under reflux conditions, as previously described (12), but with slight modifications. Subsequent to cooling, the mixture was diluted to $50 \mathrm{ml}$ with absolute methanol, sonicated $(37 \mathrm{kHz}$ ) for $2 \mathrm{~min}$ and then passed through a $0.45-\mu \mathrm{m}$ filter. Next, the hydrolyzed and non-hydrolyzed extract samples were analyzed by HPLC fractionation on a 250x4.6-mm octadecylsilane column with a 5-mm particle size (LiChrospher ${ }^{\circledR} 100$ RP-18; Merck KGaA, Darmstadt, Germany), which was attached to an Agilent HP1200 series HPLC system equipped with a G1322A quaternary pump, degasser and G1314A variable wavelength UV detector (Agilent Technologies, Inc., Santa Clara, CA, USA). Chromatographic separation was achieved with a linear gradient from $95 \%$ eluent A $[0.1 \%(\mathrm{v} / \mathrm{v})$ formic acid in water] and $5 \%$ eluent B $[0.1 \%(\mathrm{v} / \mathrm{v})$ formic acid in methanol] to a final mobile phase composed of $5 \%$ eluent $\mathrm{A}$ and $95 \%$ eluent B over $40 \mathrm{~min}$ at a flow rate of $0.8 \mathrm{ml} / \mathrm{min}$, with UV detection at 280 and $340 \mathrm{~nm}$. (+)-Catechin was purchased from Wako Pure Chemical Industries Ltd. (Osaka, Japan) and was used as the reference standard.

Chemicals. DEN (CAS no. 55-18-5; purity, >99.0\%) and $\mathrm{CCl}_{4}$ (CAS no. 56-23-5; purity, $>99.9 \%$ ) were purchased from Sigma-Aldrich Chemie GmbH (Hamburg, Germany).

Animals. All the animal procedures were performed in accordance with the guide for the care and use of laboratory animals established by the Committee for the Purpose of Control and Supervision of Experiments on Animals and the National Institutes of Health (13). In total, 40 male Wistar rats (age, 6 weeks) were obtained from the Animal House Colony of the National Research Center (Cairo, Egypt). The animals were fed a standard diet (Al Wadi Co., Giza, Egypt) and had access to water ad libitum. The rats were housed in plastic cages in an air-conditioned room at $21^{\circ} \mathrm{C}$ with a $12 \mathrm{~h}$ light/dark cycle. All the animals were allowed to acclimatize to the conditions for one week prior to the initiation of the experiments. The experimental protocol was approved by the Animal Ethics Committee of Kafrelsheikh University (Kafr el-Sheikh, Egypt).

Hepatocarcinogenesis model. The experimental protocol is summarized in Fig. 1. All the rats received an intraperitoneal (i.p.) injection of $100 \mathrm{mg} / \mathrm{kg}$ of body weight DEN dissolved in $0.9 \% \mathrm{NaCl}$ solution once per week for three weeks in order to induce hepatocarcinogenesis, as previously reported (14). The rats were then divided into four groups ( $\mathrm{n}=10$ each) one week after the final dose of DEN. The rats in group 1 received no additional treatment during the remaining 7 weeks of the study. The rats in group 2 were fed a diet that included $100 \mathrm{mg}$ C. comosum extract per $1 \mathrm{~kg}$ of food for 7 weeks. The rats in group 3 received an i.p. injection of $0.8 \mathrm{ml} / \mathrm{kg} \mathrm{CCl}$ once per week for 7 weeks in order to promote the development of DEN-induced hepatic foci and were fed a normal diet during 


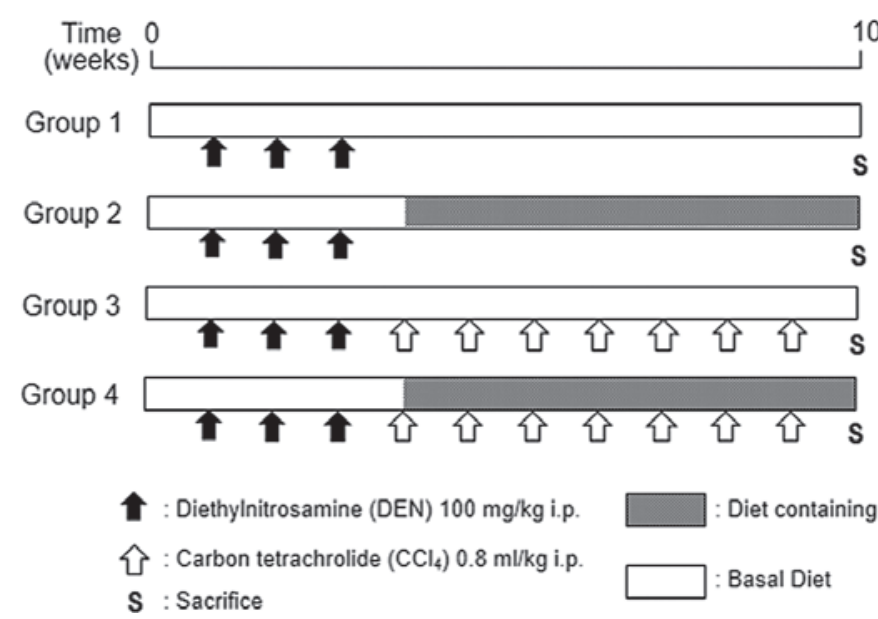

Figure 1. Experimental protocol for studying the protective activity of Calligonum comosum methanolic extract against hepatocarcinogenesis in rats.

this time period. The rats in group 4 received the same $\mathrm{CCl}_{4}$ treatment as group 3, but were fed a diet that contained $100 \mathrm{mg}$ of C. comosum extract per $1 \mathrm{~kg}$ of food for the remaining 7 weeks of the study. At the completion of the 7-week treatments, the rats were sacrificed under anesthesia, at which time the total food intake and body weights were recorded, blood samples were drawn from the caudal vena cava, and the livers were resected and weighed. The blood samples were then centrifuged at 3,000 $\mathrm{x}$ g for $15 \mathrm{~min}$, and the isolated serum was stored at $-20^{\circ} \mathrm{C}$ until analysis.

Serum biochemical assay. The serum levels of aspartate aminotransferase (AST), alanine aminotransferase (ALT) and $\gamma$-glutamyl transferase (GGT) were determined using AST/SGOT Liqui-UV ${ }^{\circledR}$, ALT/SGPT Liqui-UV ${ }^{\circledR}$ and Gamma-GT LiquiColor ${ }^{\circledR}$ kits, respectively (Stanbio Laboratory, Boerne, TX, USA).

Histological and immunohistological analyses. Liver slices obtained from the right and median lobes were fixed in $10 \%$ neutral-buffered formalin. The specimens were then dehydrated in a graded series of ethanol, cleared in xylene and embedded in paraffin wax. Next, the paraffin-embedded specimens were sectioned into $4-\mu \mathrm{m}$ samples for hematoxylin and eosin staining (Sigma-Aldrich Chemie $\mathrm{GmbH}$ ). and immunostaining. The number of oval cells was counted at high power (magnification, $\mathrm{x} 400)$ in three area fields for each rat. Immunohistochemical staining for placental glutathione $\mathrm{S}$-transferase was performed as follows. The serial sections were dewaxed, rehydrated, immersed in $0.05 \mathrm{M}$ citrate buffer $(\mathrm{pH} 6.8)$ and heated at $100^{\circ} \mathrm{C}$ for $8 \mathrm{~min}$ in an autoclave. The slides were then gently rinsed with distilled water or phosphate-buffered saline (PBS; pH 7.2) and treated with hydrogen peroxide $0.3 \%$ in absolute methanol for $30 \mathrm{~min}$ at room temperature to inactivate the endogenous peroxidase. The sections were subsequently incubated for $30 \mathrm{~min}$ with Protein Block Serum-Free (Dako, California, USA), followed by overnight at $4^{\circ} \mathrm{C}$ with a polyclonal rabbit anti-rat GST-P antibody (cat. no. 311, Medical \& Biological Laboratories Co., Ltd., Nagoya, Japan; dilution, 1:500) The sections were then incubated with a goat anti-rabbit secondary antibody (cat. no. K4003,

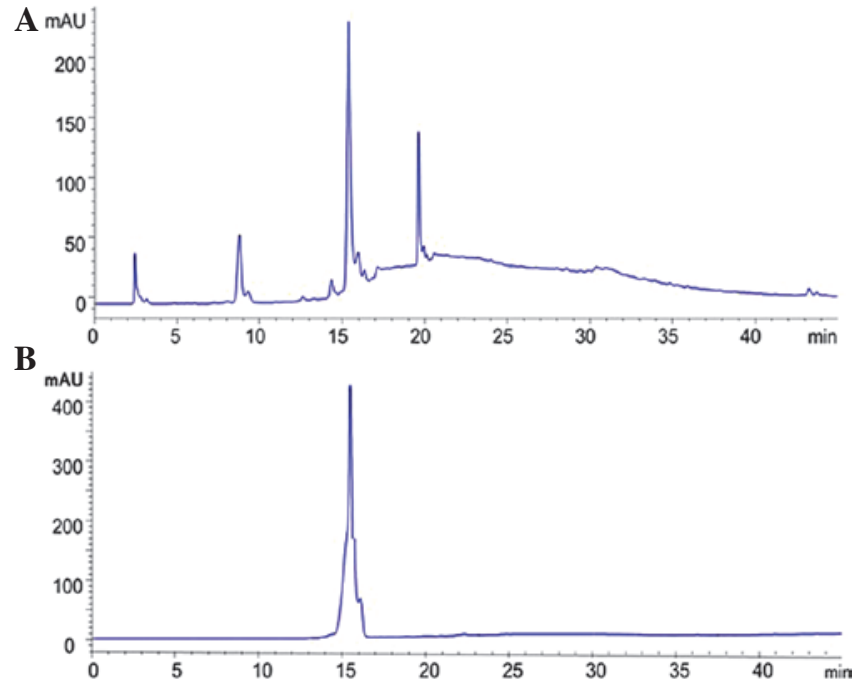

Figure 2. High-performance liquid chromatography chromatograms of (A) the hydrolyzed methanol extract of Calliogonum comosum and (B) a standard $0.8 \mu \mathrm{g} / \mathrm{ml}(+)$-catechin solution. The chromatograms were obtained under the same conditions as described in the Materials and methods section. The UV absorbance was monitored at $280 \mathrm{~nm}$.

EnVision ${ }^{\mathrm{TN}}$ System Horseradish Peroxidase Labelled Polymer; Dako) for $30 \mathrm{~min}$ at room temperature. The sections were rinsed with PBS containing 0.1\% Tween 20 (Sigma-Aldrich, St. Louis, MO, USA) between each step. Following signal detection by using a freshly prepared solution of 3,3'-diaminobenzidine tetrahydrochloride (Liquid DAB+ Substrate Chromogen System; Dako), the sections were washed in distilled water, counterstained with Mayer's hematoxylin, and dehydrated. As a negative control procedure, the primary antibody was replaced with normal rabbit serum. The analysis of GST-P-positive foci was then performed as previously described $(15,16)$.

Statistical analysis. For comparisons among different treatment groups, the homogeneity of the variance was tested using Bartlett's test that was set at a significance of 0.05 . When the samples demonstrated homogeneous variance, comparisons among treatment groups were analyzed using one-way analysis of variance, followed by Fisher's least significant difference tests. In the case that the variance was not homogeneous, the Kruskal-Wallis test was performed. The Steel-Dwass test was applied when the Kruskal-Wallis test indicated a significant difference between the groups. Data are expressed as the mean \pm standard deviation. A value of $\mathrm{P}<0.05$ was used to indicate a statistically significant difference.

\section{Results}

Phytochemical analysis

Total phenolic and flavonoid contents. The total phenolic and flavonoid contents of the methanolic $C$. comosum extract were estimated to have an activity equivalent to $335 \mathrm{mg}$ of gallic acid and $11.1 \mathrm{mg}$ of quercetin, respectively, per gram of dry extract.

DPPH radical scavenging activity. The $C$. comosum extract exhibited a dose-dependent free radical-scavenging activity in the standard DPPH assay. The concentration at which 50\% inhibition of DPPH was observed $\left(\mathrm{IC}_{50}\right)$ was $29.2 \mu \mathrm{g} / \mathrm{ml}$. 
Table I. Body and liver weights after the 7 weeks of treatment.

\begin{tabular}{|c|c|c|c|c|c|}
\hline \multirow[b]{2}{*}{ Group } & \multirow[b]{2}{*}{ Treatment } & \multirow[b]{2}{*}{ No. of rats } & \multirow[b]{2}{*}{ Body weight, $g$} & \multicolumn{2}{|c|}{ Liver weight } \\
\hline & & & & Absolute, $\mathrm{g}$ & Relative, $\%$ \\
\hline 1 & $\mathrm{DEN}$ & 10 & $223.4 \pm 39.3$ & $9.1 \pm 0.76$ & $4.14 \pm 0.55$ \\
\hline 2 & $\mathrm{DEN}+C$. comosum extract & 10 & $216.3 \pm 23.7$ & $7.67 \pm 0.57^{\mathrm{b}}$ & $3.57 \pm 0.32^{\mathrm{a}}$ \\
\hline 3 & $\mathrm{DEN}+\mathrm{CCl}_{4}$ & 10 & $194.7 \pm 13.1$ & $11.01 \pm 1.92^{\mathrm{a}}$ & $5.68 \pm 1.07^{\mathrm{a}}$ \\
\hline 4 & $\mathrm{DEN}+\mathrm{CCl}_{4}+C$. comosum extract & 10 & $218.5 \pm 17.4^{\mathrm{c}}$ & $8.64 \pm 1.12^{\mathrm{c}}$ & $3.97 \pm 0.56^{\mathrm{c}}$ \\
\hline
\end{tabular}

${ }^{\mathrm{a}} \mathrm{P}<0.05$ and ${ }^{\mathrm{b}} \mathrm{P}<0.005$, vs. group 1 ; and ${ }^{\mathrm{c}} \mathrm{P}<0.05$ vs. group 3 . Statistically significant differences were determined using Kruskal-Wallis test, followed by Steel-Dwass test. Data are expressed as the mean \pm standard deviation. $\mathrm{CCl}_{4}$, carbon tetrachloride; DEN, diethylnitrosamine; C. comosum, Calliogonum comosum.

Table II. Serum levels of AST, ALT and GGT.

\begin{tabular}{lcccc}
\hline Group & Treatment & AST, IU/l & ALT, IU/l & GGT, IU/l \\
\hline 1 & DEN & $85.36 \pm 3.57$ & $98.73 \pm 3.37$ & $20.28 \pm 3.03$ \\
2 & DEN + C. comosum extract & $55.23 \pm 5.43^{\mathrm{a}}$ & $44.75 \pm 1.72^{\mathrm{a}}$ & $13.65 \pm 2.06^{\mathrm{a}}$ \\
3 & $\mathrm{DEN}+\mathrm{CCl}_{4}$ & $102.71 \pm 4.79^{\mathrm{a}}$ & $133.08 \pm 2.99^{\mathrm{a}}$ & $35.85 \pm 2.88^{\mathrm{a}}$ \\
4 & $\mathrm{DEN}+\mathrm{CCl}_{4}+$ C. comosum extract & $64.59 \pm 3.58^{\mathrm{a}, \mathrm{b}}$ & $68.60 \pm 4.56^{\mathrm{a}, \mathrm{b}}$ & $17.91 \pm 1.23^{\mathrm{a}, \mathrm{b}}$ \\
\hline
\end{tabular}

${ }^{a} \mathrm{P}<0.005$ vs. group 1 ; and ${ }^{\mathrm{b}} \mathrm{P}<0.005$ vs. group 3 . Statistically significant differences were determined using analysis of variance, followed by Fisher's least significant difference test. Data are expressed as the mean \pm standard deviation. AST, aspartate aminotransferase; ALT, alanine aminotransferase; GGT, $\gamma$-glutamyl transferase; $\mathrm{CCl}_{4}$, carbon tetrachloride; DEN, diethylnitrosamine; C. comosum, Calligonum comosum .

HPLC profile of the methanolic extract. Whereas the HPLC profile of the non-hydrolyzed methanolic extract exhibited a number of peaks with an absorbance at $280 \mathrm{~nm}$ (data not shown), the hydrolyzed extract demonstrated only three major peaks (Fig. 1A). The most prominent peak eluted with a retention time of $15.1 \pm 0.1 \mathrm{~min}$, and was subsequently identified to be (+)-catechin by comparing with the retention time of a (+)-catechin standard solution $(0.5 \mathrm{mg} / \mathrm{ml})$. Using standard curve analysis, the amount of (+)-catechin in the extract was determined to be $113 \mathrm{mg} / \mathrm{g}$ of dry extract (Fig. 2).

\section{Biological assay}

Body and liver weights. In all the treatment groups, the rats survived throughout the entire study period with no observed severe adverse clinical effects. In addition, no significant differences in food consumption were observed among the treatment groups (data not shown). The final body and liver weights of the rats from the different treatment groups are presented in Table I. The rats treated with DEN and $\mathrm{CCl}_{4}$ (group 3) had lower body weights over the course of the study, whereas dietary supplementation with the $C$. comosum extract (group 4) was found to significantly suppress weight loss $(\mathrm{P}<0.05)$. Furthermore, the DEN-treated rats that had received $\mathrm{CCl}_{4}$ demonstrated significantly increased absolute and relative liver weights compared with the corresponding weights of the rats treated with DEN alone $(\mathrm{P}<0.05$ for absolute and relative values). Notably, supplementation with C. comosum extract significantly inhibited the increase in the absolute and relative liver weights that was induced by the
DEN and $\mathrm{CCl}_{4}$ treatment $(\mathrm{P}<0.05$; groups 3 and 4 for absolute and relative values).

C. comosum extract protects against the induction of liver enzymes. The serum levels of AST, ALT and GGT in the rats from the different treatment groups are presented in Table II. The enzyme levels were consistently and significantly lower in the rats that were fed the diet containing $C$. comosum extract (group 2) compared with the rats that were fed the standard diet (group 1; P<0.005 for AST, ALT and GGT). Compared with treatment with DEN alone (group 1), treatment with $\mathrm{CCl}_{4}$ and DEN also increased the enzyme levels (group 3; $\mathrm{P}<0.005$ for all enzymes). Furthermore, the co-administration of $C$. comosum extract (group 4) significantly suppressed the increase in liver enzyme levels compared with the levels in the rats receiving treatment with $\mathrm{CCl}_{4}$ and DEN (group 3; $\mathrm{P}<0.005$ for all enzymes).

C. comosum extract suppresses the induction of oval cell proliferation. Representative photomicrographs of the rat liver sections and the number of oval cells detected in these sections are shown in Fig. 3. Moderate oval cell proliferation was observed in the periportal region of liver sections from rats treated with DEN alone (group 1). By contrast, the proliferation was significantly reduced by supplementation with C. comosum extract (group 2; $\mathrm{P}<0.001$ ). Similarly, $\mathrm{CCl}_{4}$ treatment induced a 3.3 -fold increase in the proliferation of oval cells that infiltrated from the periportal region into the intralobular region (group 3; $\mathrm{P}<0.001$ ). However, this 
A

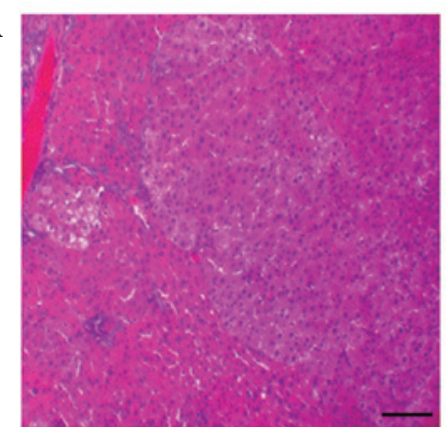

C

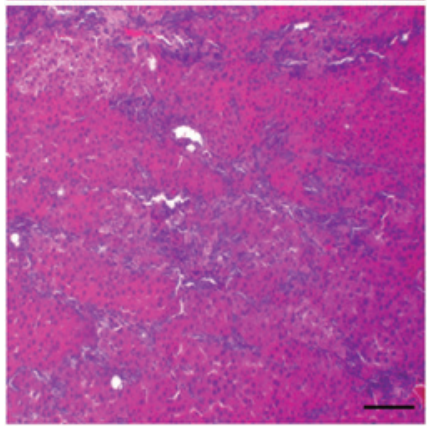

B

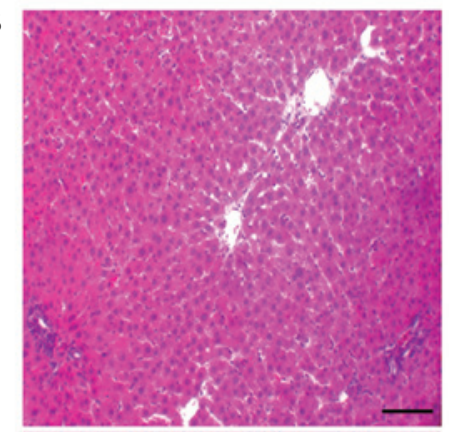

D

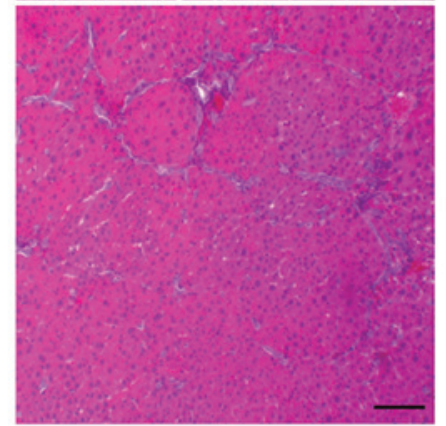

$\mathbf{E}$

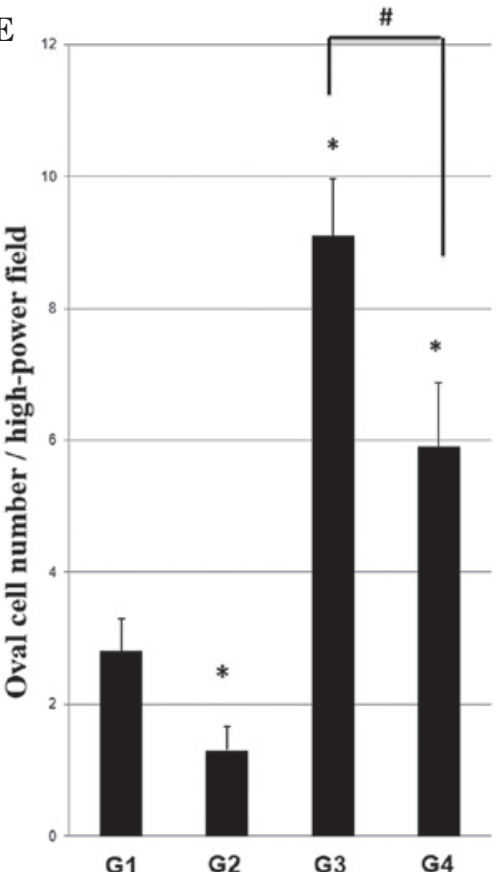

Figure 3. Histology of liver sections obtained from the rats of the different treatment groups: (A) Group 1, DEN alone; (B) group 2, DEN + C. comosum extract; (C) group 3, DEN $+\mathrm{CCl}_{4}$; and (D) group 4, DEN $+\mathrm{CCl}_{4}+$ C. comosum extract. Note the presence of oval cell proliferation and altered hepatic foci in panel (A), and marked proliferation of the oval cells that infiltrate from the periportal region to the intralobular region in panel (C). (E) Number of oval cells detected in the liver sections. Data are expressed as the mean \pm standard deviation. Statistically significant differences were determined using Kruskal-Wallis test, followed by Steel-Dwass test. " $\mathrm{P}<0.001$ vs. group 1 ; and ${ }^{~} \mathrm{P}<0.001$ vs. group 3 . DEN, diethylnitrosamine; C. comosum, Calligonum comosum; $\mathrm{CCl}_{4}$, carbon tetrachloride.
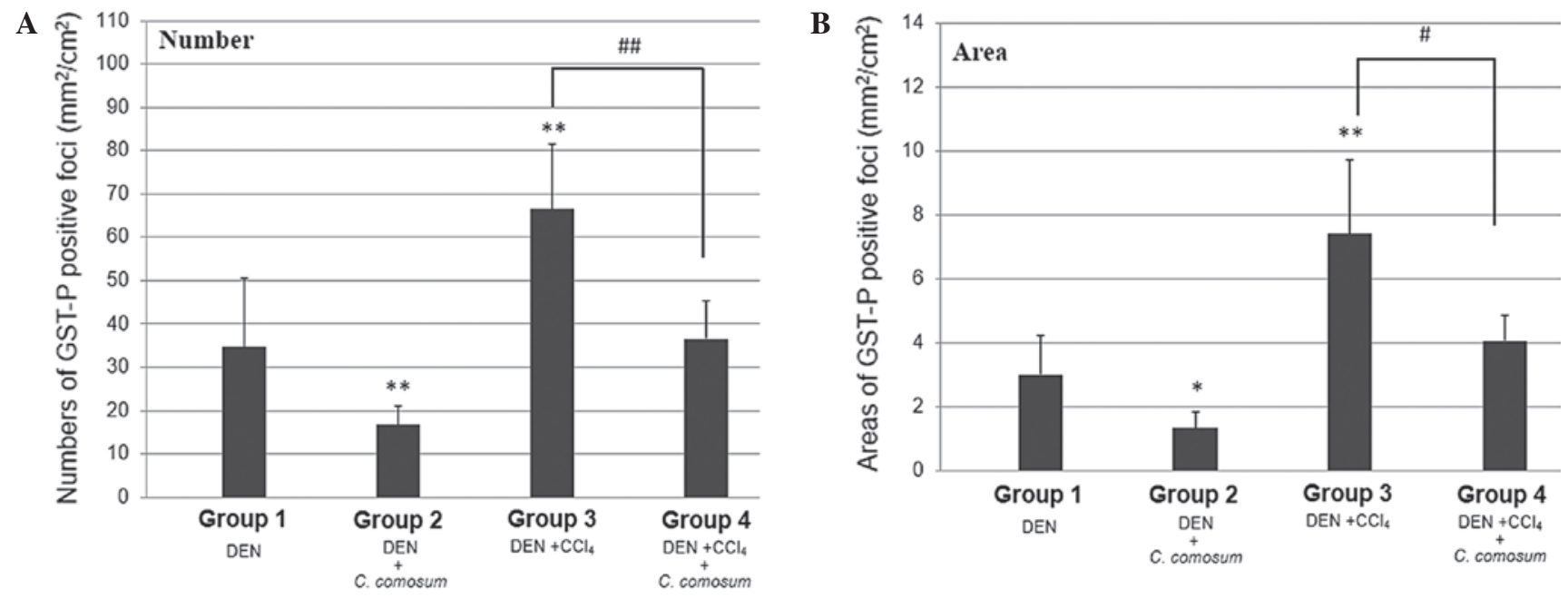

Figure 4. (A) Numbers and (B) mean areas of GST-P-positive foci $(>0.2 \mathrm{~mm}$ in diameter). Data are expressed as the mean \pm standard deviation for the 10 rats in each group. Statistically significant differences were determined using Kruskal-Wallis test, followed by Steel-Dwass test. ${ }^{*} \mathrm{P}<0.05$ and ${ }^{* *} \mathrm{P}<0.005$, vs. group 1; ${ }^{\#} \mathrm{P}<0.01$ and ${ }^{\# \#} \mathrm{P}<0.005$, vs. groups 3 and 4 . DEN, diethylnitrosamine; C. comosum, Calliogonum comosum; $\mathrm{CCl}_{4}$, carbon tetrachloride; GST-P, glutathione S-transferase placental form.

induction in proliferation was significantly suppressed by the co-administration of the C. comosum extract $(\mathrm{P}<0.001$; groups 2 and 4).

C. comosum extract suppresses the formation of GST-P-positive foci. The quantitative data obtained for the GST-P-positive foci are shown in Fig. 4. In addition, representative photomicrographs of the immunohistochemically-stained sections are shown in Fig. 5. As expected, the repeated administration of $\mathrm{CCl}_{4}$ following DEN treatment (group 3) significantly increased the number and mean area of GST-P-positive foci $(\mathrm{P}<0.005$ for number and area). By contrast, C. comosum extract significantly decreased the number and mean area of GST-P-positive foci when co-administered with DEN alone (group 2; $\mathrm{P}<0.005$ and 0.01 for number and area, respectively), as well as when DEN was combined with $\mathrm{CCl}_{4}$ (group 4; $\mathrm{P}<0.005$ and $\mathrm{P}<0.01$ for number and area, respectively).

\section{Discussion}

At present, the majority of treatments for $\mathrm{HCC}$ are not sufficiently effective, making this malignancy the second most lethal 

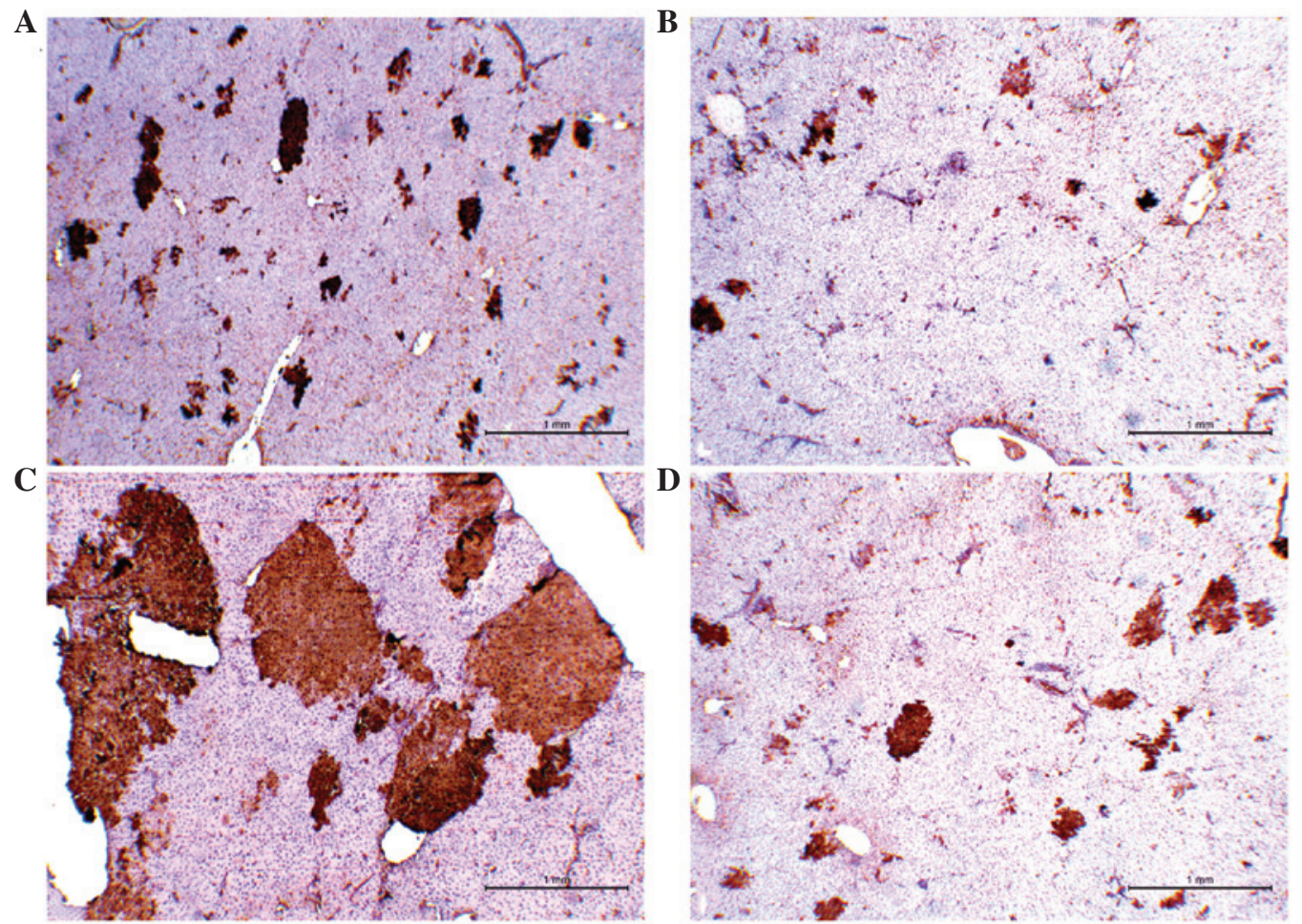

Figure 5. Immunostaining for GST-P in (A) group 1,(B) group 2, (C) group 3 and (D) group 4. Panel (A) presented GST-P positive foci. Scale bar, 1 mm. GST-P, glutathione S-transferase placental form.

type of cancer and the third leading cause of cancer-associated mortalities (1). Since the current treatment strategies are not particularly effective, chemoprevention is an important tool that can be used in order to prevent HCC (3). Chemopreventive agents isolated from plants local to areas of high HCC incidence may be particularly valuable, given their potential practical and economic benefits. A number of plant phenols, including flavonoids, are known to have HCC chemopreventive properties (3). C. comosum is a readily available Egyptian desert plant that has been reported to contain a diverse array of antioxidant flavonoids (7). The present study is the first to examine the hepatoprotective activity of $C$. comosum.

The phytochemical analysis of the methanolic C. comosum extract in the present study indicated that its phenolic content, flavonoid content and radical-scavenging activity were comparable to those of a previously reported extract obtained from plants in a different area (7). In addition, the HPLC analysis positively identified (+)-catechin in the hydrolyzed $C$. comosum extract. This result was also consistent with those previously reported for the methanolic extract and its fractions obtained from plants in a different area (7). These data suggest that $C$. comosum has a consistent phytochemical content, irrespective of the area or environment in which it is grown.

The present study also revealed that the $C$. comosum extract exhibited a chemopreventive activity against hepatocarcinogenesis induced by treatment with DEN alone or DEN in combination with $\mathrm{CCl}_{4}$. This protective effect was most notable in the suppression of proliferation of GST-P positive preneoplastic foci. The reduced serum levels of AST, ALT and GGT produced by the extract suggested hepatoprotective properties, which was confirmed by the histological evaluations of the liver sections. The protective activity of the $C$.comosum extract most likely originates from its ability to reduce damage to the liver by carcinogens, which suppress the promotion of hepatic foci induced by hepatocyte regeneration. Oval cells are not normally involved in liver regeneration, due to the regenerative capacity of hepatocytes. However, in certain conditions that cause severe liver damage, oval cells have been observed and are, therefore, considered to replenish damaged hepatocytes and bile duct cells (17). In a previous study, the proliferation of oval cells was decreased in the livers of hepatocarcinogenesis-resistant DRH strain rats (18). The presence of oval cells following DEN and $\mathrm{CCl}_{4}$ treatment is therefore indicative of severe liver damage. Thus, it is reasonable to conclude that a significant decrease in the number of oval cells in the current study indicates that the $C$. comosum extract reduced the severity of liver damage caused by $\mathrm{DEN}$ and $\mathrm{CCl}_{4}$.

The basis for the hepatoprotective effects of $C$. comosum extract may be associated with its antioxidant activity. A previous study reported that the $(+)$-catechin in a $C$. comosum extract exhibited a high antioxidant activity (7). HPLC analysis of the extract prepared in the present study also identified (+)-catechin. In addition, the extract exhibited a strong radical-scavenging activity. Catechin is a flavan-3-ol (or simply flavanol), which is a type of natural phenol and antioxidant. It is part of the chemical family of flavonoids. Epigallocatechin-3-gallate is the most abundant and potent catechin, acting as a selective inhibitor of the tyrosine phosphorylation of platelet derived growth factor receptor $\beta$ (19). In addition, it has been identified to induce apoptosis in prostate cancer cells through agonist-dependent androgen receptor (AR) activation and AR-regulated signaling pathways (20). Furthermore, catechin hydrate has been demonstrated to suppress the proliferation of different cancer 
cells, including MCF-7 human breast cancer cells through the induction of apoptosis (21) and lung cancer cell lines by the upregulation of the let-7 tumor-suppressor microRNAs (22). In addition to antioxidative properties, catechins affect the molecular mechanisms involved in carcinogenesis, including extracellular matrix degradation, angiogenesis, multidrug resistance and regulation of cell death (23). Notably, (+)-catechin can also suppress the $\mathrm{CCl}_{4}$-induced lipid peroxidation of rat livers, which is considered an important component of the mechanism by which $\mathrm{CCl}_{4}$ induces hepatocellular necrosis (24). In addition, a previous study revealed that catechins inhibited collagen production in hepatic stellate cells despite enhanced collagen transcription and suppressed collagenase activity, which indicated an antifibrogenic effect of these substances (25).

C. comosum extracts contain a number of other antioxidant flavonoids that may protect against liver damage (7). In fact, a previous study suggested that the unique combination of flavonoids in C. comosum may synergistically enhance its antioxidant activity (7). Since oxidative stress is considered to be an important component of hepatitis- and alcohol-induced hepatocarcinogenesis in humans $(1,26,27)$, the antioxidant activity of $C$. comosum extracts is a particularly promising and potentially chemoprotective agent for at-risk populations.

In conclusion, the present study revealed that a methanolic extract of C. comosum exhibited significant chemopreventive effects against DEN-induced hepatocarcinogenesis in rats. Therefore, $C$. comosum-derived formulations are promising candidates for further evaluation and the development of practical and economical chemopreventative agents in regions with high HCC prevalence.

\section{Acknowledgements}

This study was supported, in part, by a grant from the Science and Technology fund of Kafrelsheikh University, Egypt (grant no. 33-2013).

\section{References}

1. Farazi PA and DePinho RA: Hepatocellular carcinoma pathogenesis: from genes to environment. Nat Rev Cancer 6: 674-687, 2006.

2. Anwar WA, Khaled HM, Amra HA, et al: Changing pattern of hepatocellular carcinoma (HCC) and its risk factors in Egypt: possibilities for prevention. Mutat Res 659: 176-184, 2008.

3. Stagos D, Amoutzias GD, Matakos A, Spyrou A, Tsatsakis AM and Kouretas D: Chemoprevention of liver cancer by plant polyphenols. Food Chem Toxicol 50: 2155-2170, 2012.

4. Ghazanfar SA: Handbook of Arabian medicinal plants. CRC Press, Boca Raton, pp173, 1994.

5. Liu X, Zakaria MN, Islam MW, et al: Anti-inflammatory and anti-ulcer activity of Calligonum comosum in rats. Fitoterapia 72: 487-491, 2001.

6. El-Hawary Z and Kholief T: Biochemical studies on some hypoglycemic agents (II) effect of Calligonum comosum extract. Arch Pharmacal Res 13: 113-116, 1990.

7. Badria FA, Ameen M and Akl MR: Evaluation of cytotoxic compounds from Calligonum comosum L. growing in Egypt. Z Naturforsch C 62: 656-660, 2007.
8. Abdel-Sattar EA, Mouneir SM, Asaad GF and Abdallah HM: Protective effect of Calligonum comosum on haloperidol-induced oxidative stress in rat. Toxicol Ind Health 30: 147-153, 2014.

9. Singleton V and Rossi J: Colorimetry of total phenolics with phosphomolybdic-phosphotungstic acid reagents. Am J Enol Vitic 16: 144-153, 1965.

10. Lamaison JL, Petitjean-Freytet $\mathrm{C}$ and Carnat A: Rosmarinic acid, total hydroxycinnamic derivatives and antioxidant activity of Apiaceae, Borraginaceae and Lamiceae medicinals. Ann Pharm Fr 48: 103-108, 1990 (In French).

11. Braca A, De Tommasi N, Di Bari L, Pizza C, Politi M and Morelli I: Antioxidant principles from Bauhinia tarapotensis. J Nat Prod 64: 892-895, 2001.

12. Nuutila A, Kammiovirta K and Oksman-Caldentey K.M: Comparison of methods for the hydrolysis of flavonoids and phenolic acids from onion and spinach for HPLC analysis. Food Chemistry 76: 519-525, 2002.

13. Indian National Science Academy New Delhi. Guidelines for Care and Use of Animals in Scientific Research. http://icmr.nic. in/bioethics/INSA_Guidelines.pdf. Accessed October 11, 2012.

14. Puatanachokchai R, Morimura K, Wanibuchi $\mathrm{H}$, et al: Alpha-benzene hexachloride exerts hormesis in preneoplastic lesion formation of rat hepatocarcinogenesis with the possible role for hepatic detoxifying enzymes. Cancer Lett 240: 102-113, 2006.

15. Abdo W, Hirata A, Sakai H, El-Sawak A, Nikami H and Yanai T: Combined effects of organochlorine pesticides heptachlor and hexachlorobenzene on the promotion stage of hepatocarcinogenesis in rats. Food Chem Toxicol 55: 578-585, 2013.

16. Sakai H, Tsukamoto T, Yamamoto M, et al: Distinction of carcinogens from mutagens by induction of liver cell foci in a model for detection of initiation activity. Cancer Lett 188: 33-38, 2002

17. Grisham JW and Thorgeirsson SS: Liver stem cells. In: Stem cells. Potten CS (ed). Academic Press, London, pp233-282, 1997.

18. Imai K, Yamamoto M, Tanaka H, et al: Low selection of preneoplastic hepatocytes after treatment with the 2-acetylaminofluorene diet-partial hepatectomy regimen in the liver of hepatocarcinogenesis-resistant DRH strain rats. Oncol Rep 17: 55-60, 2007.

19. Ahn HY, Hadizadeh KR, Seul C, Yun YP, Vetter H and Sachinidis A: Epigallocathechin-3 gallate selectively inhibits the PDGF-BB-induced intracellular signaling transduction pathway in vascular smooth muscle cells and inhibits transformation of sis-transfected NIH 3T3 fibroblasts and human glioblastoma cells (A172). Mol Biol Cell 10: 1093-1104, 1999.

20. Lee YH, Kwak J, Choi HK, et al: EGCG suppresses prostate cancer cell growth modulating acetylation of androgen receptor by anti-histone acetyltransferase activity. Int J Mol Med 30: 69-74, 2012.

21. Alshatwi AA: Catechin hydrate suppresses MCF-7 proliferation through TP53/Caspase-mediated apoptosis. J Exp Clin Cancer Res 29: 167, 2010.

22. Zhong Z, Dong Z, Yang L, Chen X and Gong Z: Inhibition of proliferation of human lung cancer cells by green tea catechins is mediated by upregulation of let-7. Exp Ther Med 4: 267-272, 2012.

23. Demeule M, Michaud-Levesque J, Annabi B, et al: Green tea catechins as novel antitumor and antiangiogenic compounds. Curr Med Chem Anticancer Agents 2: 441-463, 2002.

24. Cholbi MR, Paya M and Alcaraz MJ: Inhibitory effects of phenolic compounds on CCl4-induced microsomal lipid peroxidation. Experientia 47: 195-199, 1991.

25. Nakamuta M, Higashi N, Kohjima M, Fukushima M, et al: Epigallocatechin-3-gallate, a polyphenol component of green tea, suppresses both collagen production and collagenase activity in hepatic stellate cells. Int J Mol Med 16: 677-681, 2005.

26. Shimoda R, Nagashima M, Sakamoto M, et al: Increased formation of oxidative DNA damage, 8-hydroxydeoxyguanosine, in human livers with chronic hepatitis. Cancer Res 54: 3171-3172, 1994.

27. McClain CJ, Hill DB, Song Z, Deaciuc I and Barve S: Monocyte activation in alcoholic liver disease. Alcohol 27: 53-61, 2002. 